\title{
A Batch Arrival Non-Markovian Queue with Three Types of Service
}

\author{
S.Maragatha Sundari \\ Research Scholar, \\ Sathyabama University \\ Asst Professor, Dept of \\ Mathematics \\ Velammal Institute of \\ Technology \\ Chennai,India
}

\author{
S.Srinivasan \\ Prof \& HOD,Dept of \\ Mathematics \\ BS.Abdur rahman University \\ Chennai,India
}

\author{
A.Ranjitham \\ Asst Professor,Dept of \\ Mathematics \\ Velammal Institute of \\ Technology \\ Chennai,India
}

\begin{abstract}
In this paper, a batch arrival non- Markovian queueing model with three types of service is considered.The customers arrive in batches of variable size.Here a single server provides three types of service, type 1 with probability $p_{1}$, type 2 with probability $p_{2}$ and type 3 with probability $p_{3}$. The service times follows general distribution. The customer may choose either type of the services. The server follows multiple vacation. Whenever the system becomes empty, the server takes a vacation and the vacation follows general distribution.On returning from vacation if the server finds no customer waiting in the system,again the server goes for vacation.The system may breakdown at random and repair time follow exponential distribution. we assume restricted admissibility of arriving batches in which not all batches are allowed to join the system at all times.The probability generating function and some probability measures of the system are also found.
\end{abstract}

\section{MATHEMATICS SUBJECT CLASSIFICATION 60K25, 60K30 KEYWORDS}

Batch arrival,Probability generating function,Random breakdown,Restricted admissibility,Multiple vacation,Mean queue size.

\section{INTRODUCTION}

Queueing problems with server vacations have been analysed by various authors with several combinations. These type of models can be found in local area networs and data communication systems etc. A $\mathrm{Mx} / \mathrm{G} / 1$ queueing model with multiple vacations is analysed by Lee et al.[1] in which arrivals occur in bulk'Chae,K.. C and Lee,H.W.[2] have analysed this bulk model with $\mathrm{N}$ - policy.Arumuganathan R. [3] analyses some bukl queueing systems with multiple vacations.For vacation models, Boo k by Takagi can be referred [4].Survey on queueing systems can be found in Doshi ,B.T [5].Lee,H.S [6]has developed a procedure to calculate the system size probabilities for a bulkqueueing model.Krishna reddy et al.[7]has analysed a bul kqueueing model with multiple vacations.Choudhry and Madan[8]proposed a bulk queueing system with restricted admissibility of arriving Batches.Thangaraj and Vanitha[9]discussed a non markovian model with two stage heterogeneous service compulsory vacation and random breakdowns.K.C.Madan,K.D.Dowman and F.A.Maraghi,[10]have studied on bulk model with restricted admissibilityand multiple vacations.S.Srinivasan and S.Maragatha sundari[11] discussed a non marovian model with three types of services and Bernoulli feed back.Kulkarni,V.G [12]discussed about the retrial queues with server subject to breakdowns and repairs .

In this model, break downs may occur at random in the steady state, At that time, it get into a repair process.Here three types of service with single server is considered.The customer can choose either type of the service.Here the server takes multiple vacation.Break down time and repair time are exponentially distributed.Service time and the vacation time is follows general distribution.

The paper is organized as follows:In section 1.1 Practical application of three types of services is discussed,in section 2, the mathematical describtion of the model is discussed.In section 3,Definitions and notations is explained.In section 4, system equations are developed for this queueing model.In section 5 , the probability generating function is obtained.In section 6 , various performance measures of the queueing system is also presented.In section 7, Conclusion and scope for further research are given.

\subsection{Practical application of three types of services}

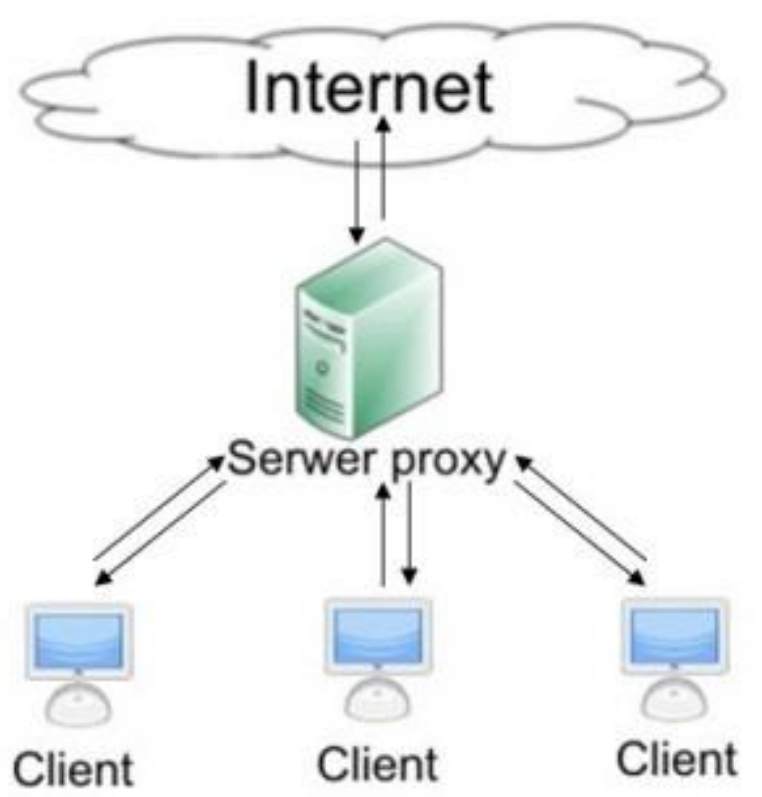


In computer networks, a proxy server is a server (a computer system or an application) that acts as an intermediary for requests from clients seeking resources from other servers. A client connects to the proxy server, requesting some service,(different types of services) such as a file, connection, web page, or other resource available from a different server and the proxy server evaluates the request as a way to simplify and control its complexity. Proxies were invented to add structure and encapsulation to distributed systems.Today, most proxies are web proxies, facilitating access to content on the World Wide Web.

Here we consider proxy server as the single server,clients are considered as types of services and server idle time is considered as the vacation period.

\section{MATHEMATICAL DESCRIBTION OF THE MODEL}

We assume the following to describe the queueing model of our study.

- Customers arrive at the system in batches of variable size in poisson process Service discipline follos 'first come first service'.Let $\lambda k_{i} d t(i=1,2 \ldots)$ be the first order probability that a batch $i$ customers arrives at the system during a short interval of time $(t, t+d t)$, where $0 \leq k_{i} \leq$ 1 and $\sum_{i=1}^{\infty} k_{i}=1$ and $\lambda>0$ is the arrival rate of batches.

- The server provides three types of service,type 1 and type 2 , and type 3 .Service time follows general distribution.Let $M_{i}(v)$ and $m_{i}(v)(i=1,2,3)$ be the distribution and the density function.

- The service time follows a general(arbitrary) distribution with distribution function $M_{i}(s)$ and density function $m_{i}(s)$.Let $\mu_{i}(x) d x$ be the conditional probability density of service completion during the interval $(x, x+d x)$, given that the elapsed time is $x$,so that

$$
\mu_{i}(x)=\frac{m_{i}(x)}{1-M_{i}(x)}, i=1,2,3
$$

And therefore

$$
m_{i}(s)=\mu_{i}(\mathrm{~s}) e^{-\int_{0}^{s} \mu_{i}(x) d x}, i=1,2,3
$$

- Here the server takes multiple vacation.

- The server's vacation follows a general (arbitrary) distribution with distribution function $V(t)$ and density function $v(t)$.Let $r(x) d x$ be the conditional probability of a completion of a vacation during the interval $(x, x+d x)$ given that the elapsed vacation time is $x$, so that

$$
\begin{aligned}
Y(x) & =\frac{v(x)}{1-V(x)} \\
& v(t)=\Upsilon(t) e^{-\int_{0}^{t} \gamma(x) d x}
\end{aligned}
$$

- The system may breakdown at random, and breakdowns are assumed to a poisson stream with mean breakdown rate $\varphi>0$.

- Further we assume that once the system breaksdown,the customer whose service is interrupted comes back to the head of the queue.Once the system breaksdown, it enters a repair process immediately.The repair times are exponentially distributed with mean repair rate $\theta>0$.

- There is a policy restricted admissibility of batches in which not all batches are allowed to join the system at all times.Let $\delta(0 \leq$ $\delta \leq 1)$ and $\varepsilon(0 \leq \varepsilon \leq 1)$ be the probability that an arriving batch will be allowed to join the system during the period of server's non-vacation period and vacation period respectively.

\section{DEFINTIONS AND NOTATIONS}

Let $N_{Q}(t)$ denote the queue size (excluding one in service) at time $t$.We introduce the random variable $Y(t)$ as follows $X(t)$

$\int 1$ if the server is busy with first type of service at time $t$ $=\left\{\begin{array}{l}2 \text { if the server is busy with second type of service at time } t \\ 3 \text { if }\end{array}\right.$ $=\{3$ if the server is busy with third type of service at time $t$ 4 if the server is on vacation at time $t$

We introduce the supplementary variable as,

$U(t)=\left\{\begin{array}{cc}M_{1}^{0}(t) \text { if } & X(t)=1 \\ M_{2}^{0}(t) \text { if } & X(t)=2 \\ M_{3}^{0}(t) \text { if } & X(t)=3 \\ V^{0}(t) \text { if } & X(t)=4\end{array}\right.$

$P_{n}^{(1)}(x, t): \operatorname{Pr}\{$ at time t,the server is active providing service and there are $n(n \geq 0)$ customers in the queue excluding the one customer in the first type of service being served and the elapsed service time for this customer is $x$ \}.

$P_{n}^{(1)}(t)=\int_{0}^{\infty} P_{n}^{(1)}(x, t) d x$ denotes the probability that at time $t$ there are $\mathrm{n}$ customers in the queue excluding one customer in the first type of service irrespective of the value of $x$.

$P_{n}^{(2)}(x, t): \operatorname{Pr}\{$ at time $t$, the server is active providing service and there are $n(n \geq 0)$ customers in the queue excluding the one customer in the second type of service being served and the elapsed service time for this customer is $x$ \}

$P_{n}^{(2)}(t)=\int_{0}^{\infty} P_{n}^{(2)}(x, t) d x$ denotes the probability that at time $\mathrm{t}$ there are $\mathrm{n}$ customers in the queue excluding one customer in the second type of service irrespective of the value of $x$.

$P_{n}^{(3)}(x, t): \operatorname{Pr}\{$ at time $t$,the server is active providing service and there are $n(n \geq 0)$ customers in the queue excluding the one customer in the third type of service being served and the elapsed service time for this customer is $x$ \}

$P_{n}^{(3)}(t)=\int_{0}^{\infty} P_{n}^{(3)}(x, t) d x$ denotes the probability that at time $\mathrm{t}$ there are $\mathrm{n}$ customers in the queue excluding one customer in the third type of service irrespective of the value of $x$.

$V_{n}(x, t): \operatorname{Pr}\{$ at time t,the server is on vacation with elapsed vacation time $x$ and there are $n(n \geq 0)$ customers in the queue\}.

$V_{n}(t)=\int_{0}^{\infty} V_{n}(x, t) d x$ denotes the probability that at time $\mathrm{t}$ there are $\mathrm{n}$ customers in the queue and the server is on vacation irrespective of the value of $x$.

$Q_{n}(t): \operatorname{Pr}$ \{at time $t$,the server is inactive due to system breakdown and the system under repair,while there are $n(n \geq$ 0 ) customers in the queue\}. 


\section{GOVERNING EQUATIONS}

The model is then,governed by the following set of differentialdifference equations:

$$
\begin{aligned}
& \frac{d}{d x} P_{0}^{(1)}(x)+\left[\lambda+\mu_{1}(x)+\varphi\right] P_{0}^{(1)}(x) \\
& =\lambda(1-\delta) P_{0}^{(1)}(x) \\
& \frac{d}{d x} P_{n}^{(1)}(x)+\left[\lambda+\mu_{1}(x)+\varphi\right] P_{n}^{(1)}(x) \\
& =\lambda(1-\delta) P_{n}^{(1)}(x)+\lambda \delta \sum_{k=1}^{n} K_{k} P_{n-k}^{(1)}(x), n \geq 1 \\
& \frac{d}{d x} P_{0}^{(2)}(x)+\left[\lambda+\mu_{2}(x)+\varphi\right] P_{0}^{(2)}(x) \\
& =\lambda(1-\delta) P_{0}^{(2)}(x) \\
& \frac{d}{d x} P_{n}^{(2)}(x)+\left[\lambda+\mu_{1}(x)+\varphi\right] P_{n}^{(2)}(x) \\
& =\lambda(1-\delta) P_{n}^{(2)}(x)+\lambda \delta \sum_{k=1}^{n} K_{k} P_{n-k}^{(2)}(x), n \geq 1 \\
& \frac{d}{d x} P_{0}^{(3)}(x)+\left[\lambda+\mu_{3}(x)+\varphi\right] P_{0}^{(3)}(x) \\
& =\lambda(1-\delta) P_{0}^{(3)}(x) \\
& \frac{d}{d x} P_{n}^{(3)}(x)+\left[\lambda+\mu_{3}(x)+\varphi\right] P_{n}^{(3)}(x) \\
& =\lambda(1-\delta) P_{n}^{(3)}(x)+\lambda \delta \sum_{k=1}^{n} K_{k} P_{n-k}^{(3)}(x), n \geq 1 \\
& \frac{d}{d x} V_{0}(x)+[\lambda+\Upsilon(x)] V_{0}(x)=\lambda(1-\varepsilon) V_{0}(x) \\
& \frac{d}{d x} V_{n}(x)+[\lambda+\Upsilon(x)] V_{n}(x) \\
& =\lambda(1-\varepsilon) V_{n}(x)+\lambda \varepsilon \sum_{k=1}^{n} K_{k} V_{n-k}(x) \\
& (\lambda+\theta) Q_{0}=0 \\
& (\lambda+\theta) Q_{n}=\lambda \sum_{k=1}^{n} K_{k} Q_{n-k}+\lambda \int_{0}^{\infty} P_{n-1}^{(1)}(x) d x \\
& +\varphi \int_{0}^{\infty} P_{n-1}^{(2)}(x) d x+\varphi \int_{0}^{\infty} P_{n-1}^{(3)}(x) d x
\end{aligned}
$$

Equations are to be solved subject to the following boundary conditions:

$$
\begin{aligned}
& P_{n}^{(1)}(0)=p_{1} \int_{0}^{\infty} \gamma(x) V_{n+1}(x) d x+p_{1} \int_{0}^{\infty} \mu_{1}(x) P_{n+1}^{(1)}(x) d x \\
& +p_{1} \int_{0}^{\infty} \mu_{2}(x) P_{n+1}^{(2)}(x) d x++p_{1} \theta R_{n+1}, n \geq 0 \\
& P_{n}^{(2)}(0)=p_{2} \int_{0}^{\infty} \Upsilon(x) V_{n+1}(x) d x+p_{2} \int_{0}^{\infty} \mu_{1}(x) P_{n+1}^{(1)}(x) d x \\
& +p_{2} \int_{0}^{\infty} \mu_{2}(x) P_{n+1}^{(2)}(x) d x++p_{2} \theta Q_{n+1}, n \geq 0 \quad(4.10 a) \\
& P_{n}^{(3)}(0)=p_{3} \int_{0}^{\infty} r(x) V_{n+1}(x) d x+p_{3} \int_{0}^{\infty} \mu_{1}(x) P_{n+1}^{(1)}(x) d x \\
& +p_{3} \int_{0}^{\infty} \mu_{3}(x) P_{n+1}^{(3)}(x) d x++p_{3} \theta Q_{n+1}, n \geq 0 \\
& V_{0}(0)=\int_{0}^{\infty} r(x) V_{0}(x) d x+\int_{0}^{\infty} \mu_{1}(x) P_{0}^{(1)}(x) d x \\
& \int_{0}^{\infty} \mu_{2}(x) P_{0}^{(2)}(x) d x+\int_{0}^{\infty} \mu_{3}(x) P_{0}^{(3)}(x) d x \theta Q_{0}
\end{aligned}
$$

$V_{n}(0)=0, n \geq 1$

\section{TIME DEPENENDENT SOLUTION}

We define the probability generating functions, $P^{(1)}(x, z)=\sum_{n=0}^{\infty} z^{n} P_{n}^{(1)}(x) ; \quad P^{(1)}(z)=\sum_{n=0}^{\infty} P_{n}^{(1)}(x),|z| \leq 1$,

$P^{(2)}(x, z)=\sum_{n=0}^{\infty} z^{n} P_{n}^{(2)}(x) ; \quad P^{(2)}(z)=\sum_{n=0}^{\infty} P_{n}^{(2)}(x),|z| \leq 1$,

$P^{(3)}(x, z)=\sum_{n=0}^{\infty} z^{n} P_{n}^{(3)}(x) ; \quad P^{(3)}(z)=\sum_{n=0}^{\infty} P_{n}^{(3)}(x),|z| \leq 1$,

$V(x, z)=\sum_{n=0}^{\infty} z^{n} V_{n}(x) ; \quad V(z)=\sum_{n=0}^{\infty} V_{n}(x) d x,|z| \leq 1$, $x>0 \quad(5.3)$

$Q(z)=\sum_{n=0}^{\infty} z^{n} Q_{n} ; \quad K(z)=\sum_{n=1}^{\infty} K_{n} z^{n}$

Now multiplying equation (4.1),(4.3) and (4.5) by suitable powers of $z$,adding (4.2),(4.4a),(4.4b) and (4.6) and summing over $\mathrm{n}$ from 0 to $\infty$ and using the generating function defined in (5.1),(5.2a),(5.2b),(5.3) and (5.4),we get

$\frac{d}{d x} P^{(1)}(x, z)$

$+\left[\lambda \delta(1-K(z))+\varphi+\mu_{1}(x)\right] P^{(1)}(x, z)=0$

$\frac{d}{d x} P^{(2)}(x, z)$

$+\left[\lambda \delta(1-K(z))+\varphi+\mu_{2}(x)\right] P^{(2)}(x, z)=0$

$\frac{d}{d x} P^{(3)}(x, z)$

$+\left[\lambda \delta(1-K(z))+\varphi+\mu_{3}(x)\right] P^{(3)}(x, z)=0$

$\frac{d}{d x} V(x, z)$

$+[\lambda \varepsilon(1-K(z))+Y(x)] V(x, z)=0$

Integrating equation (5.5),(5.6a),(5.6b) and (5.7)with respect to $x$,we have

$P^{(1)}(x, z)=P^{(1)}(0, z)\left(1-M_{1}(x)\right) e^{-Q x}$

$P^{(2)}(x, z)=P^{(2)}(0, z)\left(1-M_{2}(x)\right) e^{-Q x}$

$P^{(3)}(x, z)=P^{(3)}(0, z)\left(1-M_{3}(x)\right) e^{-Q x}$

$V(x, z)=V(0, z)(1-V(x)) e^{-T x}$

where $Q=\lambda \delta(1-K(z))+\varphi$ and $T=\lambda \varepsilon(1-K(z))$

Now multiplying equations (4.7) by suitable powers of $z$ and adding equation (4.8) summing over $n$ from 0 to $\infty$ and using equation (5.8),(5.9a) and (5.9b), we get

$\begin{aligned} & {[\lambda(1-K(z))+\theta] Q(z) } \\ = & \frac{n z}{Q}\left\{\begin{array}{r}P^{(1)}(0, z)\left[1-M_{1}^{*}(Q)\right]+P^{(2)}(0, z)\left[1-M_{2}^{*}(Q)\right] \\ +P^{(3)}(0, z)\left[1-M_{3}^{*}(Q)\right]\end{array}\right\}\end{aligned}$

Multiplying equations (4.9),(4.10a) and (4.10b) by $z^{n}$ and sum over $n$ from 0 to $\infty$, we get

$\left[z-p_{1} M_{1}^{*}(R)\right] P^{(1)}(0, z)$ 


$$
\begin{aligned}
= & p_{1} V^{*}(T) V(0, z)+p_{1} P^{(2)}(0, z) M_{2}^{*}(Q)+p_{1} P^{(3)}(0, z) M_{3}^{*}(Q) \\
& +p_{1} \theta Q(z)-p_{1} V_{0}(0) \\
{[z-} & \left.p_{2} M_{1}^{*}(Q)\right] P^{(2)}(0, z) \\
= & p_{2} V^{*}(T) V(0, z)+p_{2} P^{(2)}(0, z) M_{1}^{*}(Q)+p_{1} P^{(3)}(0, z) M_{3}^{*}(Q) \\
& +p_{2} \theta Q(z)-p_{2} V_{0}(0) \\
{[z-} & \left.p_{3} M_{3}^{*}(Q)\right] P^{(3)}(0, z) \\
= & p_{3} V^{*}(T) V(0, z)+p_{3} P^{(2)}(0, z) M_{1}^{*}(Q)+p_{1} P^{(3)}(0, z) M_{3}^{*}(Q) \\
& +p_{3} \theta Q(z)-p_{3} V_{0}(0)
\end{aligned}
$$

Multiplying equation (4.12) by $z^{n}$ and adding (4.11) and summing over $n$, we get

$V(0, z)=V_{0}(0)$

Using equation (5.12),(5.13) and (5.13a)we get

$$
\begin{aligned}
& {\left[z-p_{1} M_{1}^{*}(Q)+p_{2} M_{2}^{*}(Q)+p_{3} M_{3}^{*}(Q)\right] P^{(1)}(0, z)} \\
& =p_{1} V^{*}(T) V(0, z)+p_{1} \theta R(z)-p_{1} V_{0}(0) \\
& {\left[z-p_{1} M_{1}^{*}(Q)+p_{2} M_{2}^{*}(Q)+p_{3} M_{3}^{*}(Q)\right] P^{(2)}(0, z)} \\
& =p_{2} V^{*}(T) V(0, z)+p_{2} \delta Q(z)-p_{2} V_{0}(0)
\end{aligned}
$$

$\left[z-p_{1} M_{1}^{*}(Q)+p_{2} M_{2}^{*}(Q)+p_{3} M_{3}^{*}(Q)\right] P^{(3)}(0, z)$

$=p_{3} V^{*}(T) V(0, z)+p_{3} \delta Q(z)-p_{3} V_{0}(0)$

Using equation (5.15),(5.16) and (5.16a) in (5.11) we get

$Q(z)$

$=\frac{\varphi z\left[1-\left(p_{1} M_{1}^{*}(Q)+p_{2} M_{2}^{*}(Q)+p_{3} M_{3}^{*}(Q)\right)\right]\left[V^{*}(T)-1\right] V_{0}(0)}{D(z)}$

Where

$D(z)=Q\left[z-\left(p_{1} M_{1}^{*}(Q)+p_{2} M_{2}^{*}(Q)+p_{3} M_{3}^{*}(Q)\right)\right]$

$[\lambda(1-K(z))+\theta] \varphi z \theta\left[\begin{array}{c}1-\left(p_{1} M_{1}^{*}(Q)\right. \\ \left.+p_{2} M_{2}^{*}(Q)+p_{3} M_{3}^{*}(Q)\right)\end{array}\right]$

Using equation (5.14) and (5.17) in (5.15) (5.16) and (5.16a) we get

$P^{(1)}(0, z)=\frac{R p_{1\left[V^{*}(T)-1\right][\lambda(1-k(z))+\theta] V_{0}(0)}}{D(z)}$

$P^{(2)}(0, z)=\frac{R p_{2\left[V^{*}(T)-1\right][\lambda(1-k(z))+\theta] V_{0}(0)}}{D(z)}$

$P^{(3)}(0, z)=\frac{R p_{3\left[V^{*}(T)-1\right][\lambda(1-k(z))+\theta] V_{0}(0)}}{D(z)}$

After integrating equation (5.8) with respect to $x$, we have

$P^{(1)}(z)=\frac{Q p_{1\left[V^{*}(T)-1\right][\lambda(1-k(z))+\theta]\left[1-M_{1}^{*}(Q)\right] V_{0}(0)}}{D(z)}$

Where $M_{1}^{*}(Q)=\int_{0}^{\infty} e^{-Q x} d M_{1}(x)$ is the Laplace transform of first phase of service time.

After integrating equation (5.9) with respect to $x$, we have

$P^{(2)}(z)$

$=\frac{Q p_{2\left[V^{*}(T)-1\right][\lambda(1-k(z))+\theta]\left[1-M_{2}^{*}(Q)\right] V_{0}(0)}^{D(z)}}{\text { ( })}$

Where $M_{2}^{*}(Q)=\int_{0}^{\infty} e^{-Q x} d M_{2}(x)$ is the Laplace transform of second phase of service time.

After integrating equation (5.9a) with respect to $x$, we have $P^{(3)}(z)$

$=\frac{Q p_{3\left[V^{*}(T)-1\right][\lambda(1-k(z))+\theta]\left[1-M_{3}^{*}(Q)\right] V_{0}(0)}^{D(z)}}{\text { (z) }}$

Where $M_{3}^{*}(Q)=\int_{0}^{\infty} e^{-Q x} d M_{3}(x)$ is the Laplace transform of third phase of service time.

After integrating equation (5.10) with respect to $x$, we have
$V(z)=\frac{\left[1-V^{*}(T)\right]}{T} V_{0}(0)$

Where $V^{*}(T)=\int_{0}^{\infty} e^{-T x} d V(x)$ is the Laplace transform of vacation time.

In order to determine

$P^{(1)}(z), P^{(2)}(z), P^{(3)}(z), V(z), Q(z)$ completely.we have yet to determine the unknown $V_{0}(0)$ which appears in the numerator of the right sides of equations (5.17),(5.21),(5.22) and (5.22a) and (5.23).For that purpose,we shall use the normalizing condition

$P^{(1)}(1)+P^{(2)}(1)+P^{(3)}(1)+V(1)+Q(1)=1$

$P^{(1)}(1)$

$=\frac{-\lambda \theta \varepsilon p_{1} K^{\prime}(1)\left(1-1-M_{1}^{*}(\varphi)\right) V^{*}(0)}{d r} V_{0}(0)$

$P^{(2)}(1)$

$=\frac{-\lambda \theta \varepsilon p_{2} K^{\prime}(1)\left(1-1-M_{2}^{*}(\varphi)\right) V^{*}(0)}{d r} V_{0}(0)$

$\left(5 . P^{(3)}\right)(1)$
$\quad=\frac{-\lambda \theta \varepsilon p_{3} K^{\prime}(1)\left(1-1-M_{3}^{*}(\varphi)\right) V^{*}(0)}{d r} V_{0}(0)$

$Q(1)$

$=\frac{-\lambda \varphi \varepsilon K^{\prime}(1)\left(1-\left(p_{1} M_{1}^{*}(\varphi)+p_{2} M_{2}^{*}(\varphi)+p_{3} M_{3}^{*}(\varphi)\right)\right) V^{*}(0)}{d r}$

$\times V_{0}(0)$

$V(1)=) V^{*}(0) V_{0}(0)$

Where $d r=\left(1-\left(p_{1} M_{1}^{*}(\varphi)+p_{2} M_{2}^{*}(\varphi)+p_{3} M_{3}^{*}(\varphi)\right)\right)$

$$
\left.\left[-\lambda K^{\prime}(1)(\delta \theta+\varphi)-\varphi \theta\right]+\varphi \theta\right)
$$

$P^{(1)}(1), P^{(2)}(1), P^{(3)}(1), V(1), R(1)$ denote the steady state probabilities that the server is providing first type of service,second type of service and third type of service,server on vacation and server under repair without regard to the number of customers $\mathrm{n}$ the queue. Now using equation (5.25),(5.26),(5.26a),(5.27) and (5.28) into the normalizing condition (5.24) and simplifying, we obatain

$V_{0}(0)$

$=\frac{\varphi \theta-\left[\lambda K^{\prime}(1)(\delta \theta+\varphi)\right]\left[1-\left(p_{1} M_{1}^{*}(\varphi)+p_{2} M_{2}^{*}(\varphi)+p_{3} M_{3}^{*}(\varphi)\right]\right.}{d r}$

$\left.d r_{1}=\right) V^{*}(0)\left[1-\left(p_{1} M_{1}^{*}(\varphi)+p_{2} M_{2}^{*}(\varphi)+p_{3} M_{3}^{*}(\varphi)\right]\right.$

$\left.\left[\lambda M^{\prime}(1)(\theta(\delta-\varepsilon)+\varphi(1-\varepsilon))+\varphi \beta\right]-\varphi \theta\right) V^{*}(0)$ and hence the utilization factor $\rho$ of the system is given by

$$
\begin{gathered}
\rho \varepsilon(\theta+\varphi) K^{\prime}(1)\left[1-\left(p_{1} M_{1}^{*}(\varphi)+p_{2} M_{2}^{*}(\varphi)+p_{3} M_{3}^{*}(\varphi)\right]\right. \\
\varphi \theta-\left[1-\left(p_{1} M_{1}^{*}(\varphi)+p_{2} M_{2}^{*}(\varphi)+p_{3} M_{3}^{*}(\varphi)\right]\left[\lambda K^{\prime}(1)(\theta(\alpha-\varepsilon)\right.\right. \\
+\varphi(1-\varepsilon))+\varphi \theta]
\end{gathered}
$$

Where $\rho<1$ is the stability condition under which the steady state exists.Let $P_{q}(z)$ denote the probability generating function 
of the queue.size irrespective of the server state.Then adding equations (5.17),(5.21),(5.22) and (5.22a) we obtain

$$
\begin{aligned}
& P_{q}(z)=P^{(1)}(z)+P^{(2)}(z)+P^{(3)}(z)+V(z)+R(z) \\
& P_{q}(z)=\frac{N(z)}{D(z)}+\frac{\left[1-V^{*}(T)\right]}{T} V_{0}(0) \\
& N(z)=\left[V^{*}(T)-1\right] \\
& \left.\left[1-\left(p_{1} M_{1}^{*}(Q)+p_{2} M_{2}^{*}(Q)+p_{3} M_{3}^{*}(Q)\right)\right)\right] \\
& {\left[\lambda\left(1-K^{\prime}(1)\right)+\theta+\varphi z\right] V_{0}(0)}
\end{aligned}
$$

And $D(z)$ is given by in the equation (5.18).Substituting for $V_{0}(0)$ from (5.29) into (5.31).we have explicitly determined the probability generating function of the queue size.

\section{THE MEAN QUEUE SIZE AND THE MEAN SYSTEM SIZE}

Let $L_{q}$ denote the mean number of customers in the queue under the steady state. Then we have

$$
\begin{aligned}
& L_{q}=\frac{d}{d z} P_{q}(z) a t z=1 \\
& L_{q}=\lim _{z \rightarrow 1} \frac{D^{\prime}(1) N^{\prime \prime}(1)-N^{\prime}(1) D^{\prime \prime}(1)}{2 D^{\prime}(1)^{2}} V_{0}(0) \\
& \quad+\frac{\lambda \varepsilon K^{\prime}(1) V^{* \prime \prime}(0)}{2} V_{0}(0)
\end{aligned}
$$

Where primes and double primes in (6.1) denote first and second derivative at $z=1$, respectively.Carrying out the derivative at $z=1$ we have

$$
\begin{gathered}
\left.N^{\prime}(1)=-\lambda \varepsilon K^{\prime}(1)(\theta+\varphi)\right) V^{* \prime}(0)\left[p_{1} M_{1}^{*}(\varphi)+p_{2} M_{2}^{*}(\varphi)\right. \\
\left.+p_{3} M_{3}^{*}(\varphi)\right]
\end{gathered}
$$

$$
\begin{aligned}
& N^{\prime \prime}(1)=\left[1-p_{1} M_{1}^{*}(\varphi)+p_{2} M_{2}^{*}(\varphi)+p_{3} M_{3}^{*}(\varphi)\right] \\
& {\left[\lambda^{2} \varepsilon^{2}\left(K^{\prime}(1)\right)^{2}(\theta+\varphi)\right) V^{* \prime \prime}(0)-\lambda \varepsilon V^{*^{\prime}}(0)(\theta+\varphi) K^{\prime \prime}(1)} \\
& \left.+2 K^{\prime}(1)\left(\varphi-\lambda K^{\prime}(1)\right)\right]-2 \lambda^{2} \delta \varepsilon\left(K^{\prime}(1)\right)^{2}(\theta+\varphi) V^{* \prime}(0) \\
& {\left[p_{1} M_{1}^{*}(\varphi)+p_{2} M_{2}^{*}(\varphi)+p_{3} M_{3}^{*}(\varphi)\right]} \\
& D^{\prime}(1)=\varphi \theta-\left[\lambda K^{\prime}(1)(\delta \theta+\varphi)+\varphi \theta\right][1 \\
& -\left(p_{1} M_{1}^{*}(\varphi)+p_{2} M_{2}^{*}(\varphi)\right. \\
& \left.+p_{3} M_{3}^{*}(\varphi)\right]
\end{aligned}
$$$$
\left[\lambda^{2} \varepsilon^{2}\left(K^{\prime}(1)\right)^{2}(\theta+\varphi)\right) V^{* \prime \prime}(0)-\lambda \varepsilon V^{*^{\prime}}(0)(\theta+\varphi) K^{\prime \prime}(1)
$$

$$
\begin{gathered}
D^{\prime \prime}(1)=\left[1-\left(p_{1} M_{1}^{*}(\varphi)+p_{2} M_{2}^{*}(\varphi)+p_{3} M_{3}^{*}(\varphi)\right)\right] \\
{\left[-\lambda K^{\prime \prime}(1)(\delta \theta+\varphi)+2 \lambda^{2} \delta\left(K^{\prime}(1)\right)^{2}\right]-2 \lambda(\delta \theta+\varphi) K^{\prime}(1)} \\
{\left[1+\left(p_{1} M_{1}^{*}(\varphi)+p_{2} M_{2}^{*}(\varphi)+p_{3} M_{3}^{*}(\varphi)\right)\right]-2 \lambda \delta \theta K^{\prime}(1)} \\
\left(p_{1} M_{1}^{*}(\varphi)+p_{2} M_{2}^{*}(\varphi)+p_{3} M_{3}^{*}(\varphi)\right)
\end{gathered}
$$

Then if we substitute the values from (6.2),(6.3),(6.4) and (6.5) into (6.1) we obtain $L_{q}$ in the closed form.Further we find the mean system size $L$ using Little's formulae. Thus we have

$L=L_{q}+\rho$

Where $L_{q}$ has been found by equation (6.1) and $\rho$ is obtained from equation (5.30).

\section{CONCLUSION}

In this paper a batch arrival queuing model with three types of services with restricted admissibility, breakdowns and multiple vacations is discussed.This model is very much useful in communication network areas and large scale production industries.As a future work, this model can be extended to batch arrival with multi types of services.

\section{REFRENCES}

[1] Lee,H.W.,Lee,S.S,.Park,J.O, and Chae,K.C.,Analysis of the $\mathrm{M}^{[\mathrm{x}]} / \mathrm{G} / 1$ queue with N-policy and multiple vacations,J.Appl.Prob, 31 (1994),476-496.

[2] Chae,K.C. and Lee,H.W., $\mathrm{M}^{[\mathrm{x}]} / \mathrm{G} / 1$ vacation models with N-policy: Heuristic interpretation of mean waiting time,J.Ops.Res.Soc,46 (1995),1014-1022.

[3] Arumaganathan,R..,Bulk queueing model with server failures and multiple vacations,Ph.D. Thesis,Bharathiyar University,Coimbatore, 1997.

[4] Takagi,H.,Queueing analysis :A founadation of Performance Evaluation, Vol 1:Vacations and priority Systems,Part-1,North Holland,1991.

[5] Doshi,B.T.,Queuing Systems with vacations:A survey,Queueing Systems ,1 (1986),(29-66)

[6] Lee., H.S., Steady state probabilities for the server vacation model with group arrivals under control operation policy,J.Korean OR/MS Soc, 16 (1991),36-48

[7] Krishna Reddy,G.V., Nadarajan,R. and Armuganathan,R.,Analysis of a bulk queue with N-policy multiple vacations and set up times,Computers Ops Res, 25 (1998),957-967.

[8] G.Choudhury and K.C.Madan ,A batch arrival Bernoulli vacation queue with random set up time under restricted admissibility policy,International Journal of Operations Research,USA,2 (2007),81-97.

[9] V.Thangaraj and S.Vanitha M/G/1 Queue with Two-Stage heterogeneous service compulsory server vacation and random breakdowns Int.J.Contemp.Math.Sciences,5 (2010),307-322.

[10] F.A.Maraghi,K.C.Madan and K.D.Dowman,Batch arrival queueing system with random breadowns and Bernoulli schedule server vacations having general time distribution,International Journal of Information and Management Sciences, 20 (2009),55-70.

[11] S.Maragathasundari and S.Srinivasan Transient analysis of M/G/1 queue with Bernoulli feedback and three types of service,International conference on Mathematical modeling and Applied Soft computing,CIT,Coimbatore,India 2 (2012).

[12] V.G.Kulkarni and B.D.Choi,Retrial queues with server subject to breakdowns and repairs,Queueing Systems,7 (1990),191-209. 